\title{
COMMENT
}

\section{A pulmonary ILC3 niche promotes neonatal mucosal immunity to respiratory bacterial infection and is associated with postnatal lung development}

\author{
Malcolm R. Starkey (iD) ${ }^{1,2}$ \\ Mucosal Immunology (2020) 13:385-387; https://doi.org/10.1038/s41385-020-0283-9
}

The importance of innate lymphoid cell (ILC) interactions with stromal cells in discrete tissues niches is emerging as a key regulator of homeostasis and repair. A recent study by Oherle et al., ${ }^{1}$ extends this phenomenon to developmental processes, showing that ILC progenitors seed the neonatal lung and respond to alveolar fibroblast-derived insulin-like growth factor 1 (IGF1) to promote group 3 ILC (ILC3) biogenesis linking postnatal lung maturation with the development of mature pulmonary ILC3 and lung mucosal defenses. They show that premature human infants and mice with experimental bronchopulmonary dysplasia (BPD), have reduced IGF1 and ILC3 resulting in increased susceptibility to early life Streptococcus pneumoniae (S. pneumoniae) bacterial infection.

\section{ILC3 ARE ESSENTIAL FOR HOST DEFENSE AGAINST RESPIRATORY BACTERIAL INFECTION IN THE NEWBORN}

Newborns have immature mucosal defenses that contribute to increased susceptibility to infection with respiratory bacterial pathogens. Exposure of neonatal mice to commensal bacteria immediately after birth is required for effective host defense against $S$. pneumoniae, which is mediated by interleukin (IL)- $22^{+}$ ILC3 influx into the lungs of neonatal mice. ${ }^{2}$ Extending on this seminal work, Oherle et al., ${ }^{1}$ show that neonatal $1122^{\mathrm{f} / \mathrm{f}}$ Rorc ${ }^{\mathrm{Cre} /+}$ mice, that lack IL-22 production in RAR-related orphan receptor (RORY, encoded by Rorc) $\mathrm{t}^{+}$ILC and T helper (Th)-22 cells, were more susceptible to early life respiratory $S$. pneumoniae infection, with neonatal mice succumbing to infection within 5-6 days. Intratracheal administration of mouse recombinant IL-22, significantly increased survival rates post infection. This appeared to be dependent on IL-22 ${ }^{+}$ILC3 as intratracheal adoptive transfer of sorted $\mathrm{CD}_{4} 5^{+}$Lineage $^{-} \mathrm{RORYt}^{+}$ILCs directly into the lungs protected against infection-induced death. Intratracheal administration of recombinant cytokines and immune cells to neonates is a unique technical advance, ensuring delivery to the lower airways and parenchyma that may not be achieved by classically used intranasal or intraperitoneal routes of administration. Recent evidence links neuropeptides, intrinsic cellular rhythms and synergy with cyclic patterns of food intake to mucosal immunity regulated by ILC3 activity. ${ }^{3}$ The impact of circadian rhythms and food intake on IL-22 production and ILC3 maturation in the developing neonatal lung and in promoting local lung immunity to respiratory bacterial pathogens remains to be elucidated. In addition, the impacts of early life ILC3 priming by infection or premature birth on susceptibility to chronic lung diseases in laterlife is unknown and requires further exploration.

\section{ILC PRECURSORS GIVE RISE TO MATURE ILC3 IN THE NEONATAL LUNG AND PROTECT AGAINST RESPIRATORY S. PNEUMONIAE INFECTION}

It has been shown previously that pulmonary ILC subsets, particularly group 2 ILC (ILC2), expand rapidly in the developing postnatal mouse lung. ${ }^{4,5}$ These signals involve IL-33 release from stromal cells driving local ILC2 expansion., 4,5 ILC2 are more abundant in the neonatal lung and resident neonatal ILC2 are reported to give rise to long-lived ILC2 in the adult lung. ${ }^{4-7}$ There is also substantial plasticity of pulmonary ILC2, with their conversion to ILC3 in the lung observed in adult mice under inflammatory conditions. ${ }^{5}$ However, the dynamics of lung specific ILC progenitors in the neonatal lung and their capacity to differentiate into mature lung resident ILC3 was unknown. Oherle et al., show that promyelocytic leukemia zinc finger protein (PLZF, encoded by Zbtb16) ${ }^{+}$ILC precursors seed the lung after birth and peak after one week before declining thereafter (Fig. 1a). The appearance of mature lung ILC3 is slightly delayed and peaks at postnatal week 2. The authors block development of ILC3, by using lung specific diphtheria toxoid-mediated depletion of ZBTB16 $^{+}$ILC precursors, which promoted susceptibility to respiratory S. pneumoniae infection in early life. ${ }^{1}$ Comparatively, adoptive transfer of ILC precursors into neonatal mouse lungs protected against infection-induced death (Fig. 1b).

\section{ALVEOLAR STROMAL CELLS CREATE A LOCAL MICROENVIRONMENT TO SUPPORT ILC3 PROLIFERATION AND MATURATION AND IGF1 IS CRITICAL FOR ESTABLISHMENT OF NEONATAL PULMONARY ILC3 NICHE}

Adventitial stromal cells in multiple organs, including the lung, have been shown to define ILC2 tissue niches. ${ }^{8}$ This has been particularly well characterized in adult mouse tissues. However, characterization of lung resident tissue niches for ILC progenitors and ILC3, particularly in the neonatal lung had not been explored previously. In their study, Oherle et al., ${ }^{1}$ show using confocal imaging that $\mathrm{ZBTB}^{+} 6^{+} \mathrm{ILC}$ precursors localize to platelet-derived growth factor (PDGFR) $a^{+}$alveolar fibroblasts in the neonatal lung.

\footnotetext{
${ }^{1}$ Department of Immunology and Pathology, Central Clinical School, Monash University, Melbourne, VIC, Australia and ${ }^{2}$ Priority Research Centre's GrowUpWell and Healthy Lungs, School of Biomedical Sciences and Pharmacy, The University of Newcastle and Hunter Medical Research Institute, New Lambton Heights, NSW, Australia

Correspondence: Malcolm R. Starkey (malcolm.starkey@monash.edu)
}

Received: 2 March 2020 Accepted: 3 March 2020

Published online: 17 March 2020 


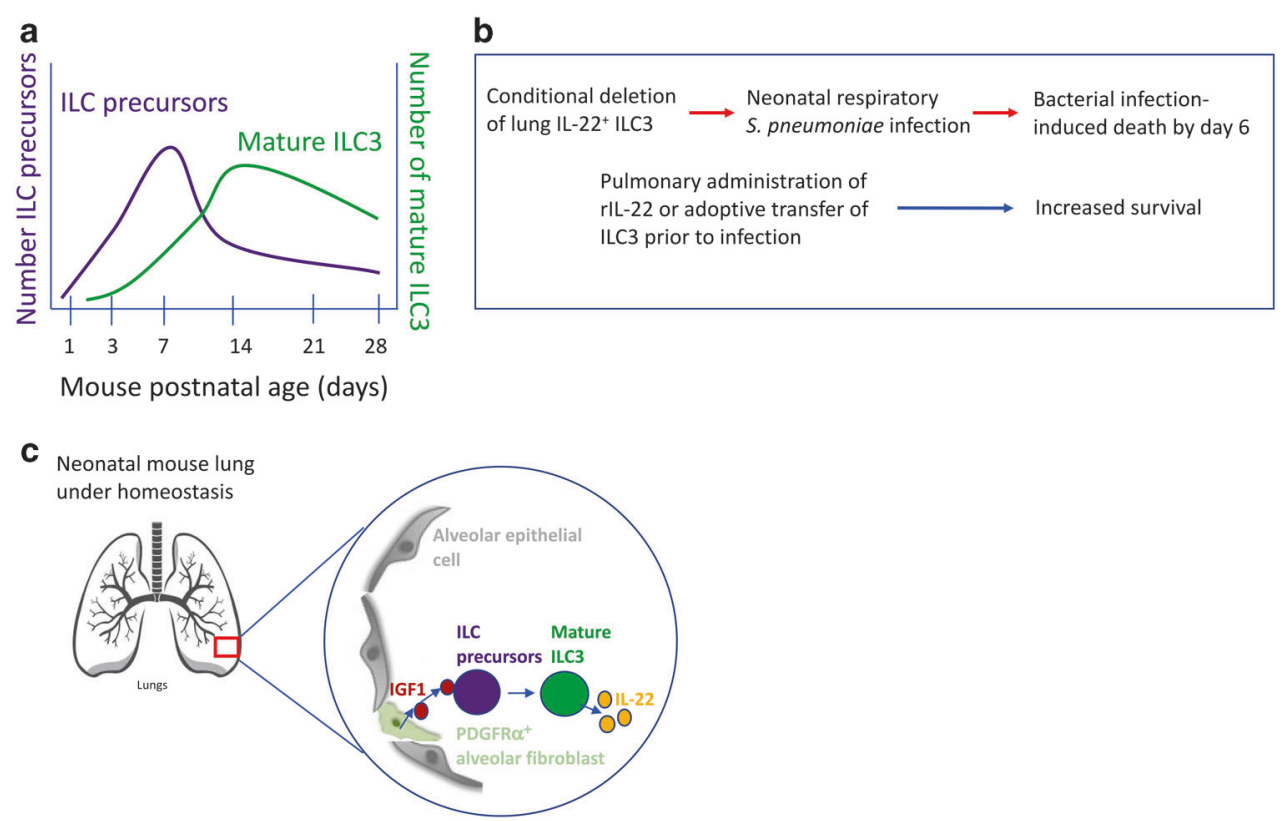

Fig. 1 Innate lymphoid cell (ILC) progenitors seed the neonatal lung and give rise to mature pulmonary group 3 ILC (ILC3) to promote local mucosal immunity to bacterial pathogens. a ILC precursors seed the neonatal lung within the first postnatal week and give rise to resident mature pulmonary ILC3. b Loss of ILC progenitors and interleukin (IL)-22 ${ }^{+}$ILC3 in the neonatal lung increases susceptibility to early life Streptococcus pneumoniae (S. pneumoniae) infection. c Neonatal lung ILC precursors are located in close proximity to platelet-derived growth factor receptor A (PDGFR $\alpha)^{+}$fibroblasts that produce insulin-like growth factor 1 (IGF1) and drive local ILC3 proliferation.

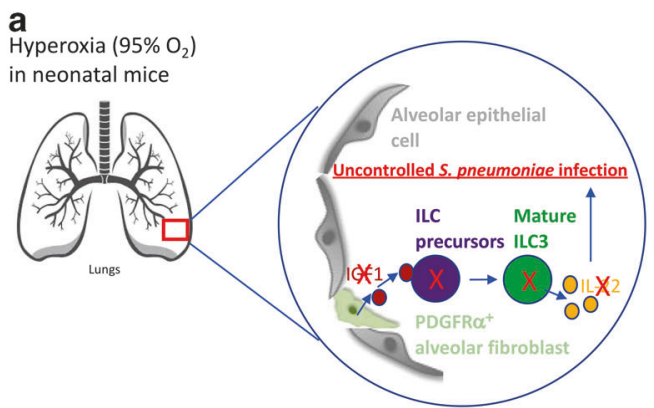

b Human newborn with BPD

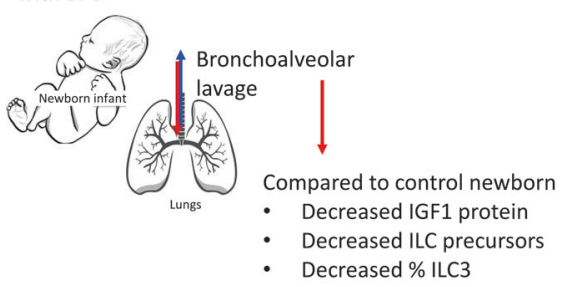

Fig. 2 Loss of neonatal pulmonary group 3 innate lymphoid cells (ILC3) is associated with impaired lung development. a Experimental bronchopulmonary dysplasia (BPD) induced by 1 week of $95 \% \mathrm{O}_{2}$ exposure results in decreased pulmonary ILC precursors, ILC3 and insulinlike growth factor 1 (IGF1) fibroblasts in the lungs of neonatal mice. b Human newborns with BPD have decreased protein levels of IGF1 and percentage of ILC3 in bronchoalveolar lavage fluid (BALF) compared to controls.

These PDGFRa ${ }^{+}$alveolar fibroblasts created a microenvironment essential for the proliferation of ILC3 from lung resident ILC precursors (Fig. 1c). The paracrine factors produced by alveolar fibroblasts that support expansion and maturation of ILC precursors was assessed by mass spectrometry. Through this strategy, Oherle et al., identified growth factor signaling pathways and IGF1 as the crucial regulator of ILC precursor expansion and ILC3 maturation. This was confirmed in ex vivo cell culture experiments combining mouse alveolar aggregates with ILC progenitors. Co-culture of mouse alveolar aggregates with ILC progenitors led to increased proliferation of ILC progenitors and increased numbers of mature ILC3. The proliferation of progenitors and ILC3 differentiation could be blocked by the addition of anti-IGF1 into the co-culture system. This was then confirmed in vivo, by tamoxifen-mediated depletion of IGF1 in neonatal mice that resulted in a loss of lung ZBTB16 ${ }^{+}$ILC precursors and impaired ILC3 maturation. Depletion of IGF1 also predisposed mice to respiratory $S$. pneumoniae infection and adoptive transfer of ILC3 was again protective against infection-induced death. ${ }^{1}$ These data highlight that $\mathrm{PDGFRa}^{+}$alveolar fibroblast-derived
IGF1 is crucial for generating local mature lung ILC3 that are protective against neonatal respiratory bacterial infection.

\section{BPD IS ASSOCIATED WITH A LOSS OF LUNG ILC3 AND DECREASED IGF1 LEVELS-A POTENTIAL LINK TO IMPAIRED LUNG DEVELOPMENT}

Using an experimental mouse model of BPD, induced by exposure of neonatal mice to $95 \% \mathrm{O}_{2}$ for the first week after birth, Oherle et al., show that exposure to hyperoxia caused a reduction in the number of IGF1 ${ }^{+}$cells in the alveolar space and decreased the number of proliferating ILC progenitors as well as the total number of lung resident mature ILC3 (Fig. 2a). Mice with experimental BPD had increased susceptibility to bacterial infection and adoptive transfer of ILC3 into the neonatal lung protected against respiratory $S$. pneumoniae infection-induced death. The clinical relevance of this observation was then confirmed in human infants with BPD (Fig. 2b). Infants with BPD had decreased levels of IGF1 protein and reduced numbers of ILC3 in the bronchoalveolar lavage compared to non-BPD infant 
controls. ${ }^{1}$ Whilst these studies show an association between a loss in IGF1 and ILC3, and a condition known to be associated with impaired lung development, there is currently no definitive evidence that a loss of ILC3 in the neonatal period results in long-term aberrant changes in lung structure and function.

\section{CONCLUSION AND KEY FUTURE DIRECTIONS FOR THE FIELD}

S. pneumoniae is a common bacterial pathogen causing respiratory infection in newborn infants. However, there are a number of additional respiratory pathogens, both bacterial (e.g., nontypeable Hemophilus Influenzae) and viral (e.g., respiratory syncytial virus, rhinovirus) that may be influenced by developmental regulation of ILC precursors and local ILC3 maturation in the neonatal lung. Exploration of a range of pathogens as well as environmental challenges (e.g., pollution) on ILC-mediated lung developmental processes is warranted. Whilst an association between a loss in pulmonary ILC3 and BPD, a human condition known to be linked to impaired lung development, has been initially explored, the implications of ILC-driven aberrant lung development on long-term pulmonary mucosal defenses to microbial insults, lung function and susceptibility to chronic respiratory diseases in later-life remains to be fully elucidated.

\section{ACKNOWLEDGEMENTS}

M.R.S. is supported by an Australian Research Council Discovery Early Career Researcher Award Fellowship, National Health and Medical Research Council of Australia Project Grants, Thoracic Society of Australia and New Zealand Lungs for Life
Grant, The University of Newcastle, The Hunter Medical Research Institute (HMRI) and Monash University.

\section{ADDITIONAL INFORMATION}

Competing interests: M.R.S. has no relevant conflicts of interest to declare.

Publisher's note Springer Nature remains neutral with regard to jurisdictional claims in published maps and institutional affiliations.

\section{REFERENCES}

1. Oherle, K. et al. Insulin-like growth factor 1 supports a pulmonary niche that promotes type 3 innate lymphoid cell development in newborn lungs. Immunity 52, 275-294 (2020).

2. Gray J. Oehrle K. Worthen G. Alenghat T. Whitsett J. Deshmukh H. Intestinal commensal bacteria mediate lung mucosal immunity and promote resistance of newborn mice to infection. Sci. Transl. Med. 9, eaaf9412 (2017).

3. Seillet, $C$. et al. The neuropeptide VIP confers anticipatory mucosal immunity by regulating ILC3 activity. Nat. Immunol. 21, 168-177 (2020).

4. Saluzzo, S. et al. First-breath-induced type 2 pathways shape the lung immune environment. Cell Rep. 18, 1893-1905 (2017).

5. Starkey M.R., McKenzie AN., Belz GT., Hansbro P.M. Pulmonary group 2 innate lymphoid cells: surprises and challenges. Mucosal Immunol. 12, 299-311 (2019).

6. Schneider, C. et al. Tissue-resident group 2 innate lymphoid cells differentiate by layered ontogeny and in situ perinatal priming. Immunity $\mathbf{5 0}$, 1425-1438 (2019)

7. Loering S., Cameron G.J.M., Starkey M.R., Hansbro P.M. Lung development and the emerging roles for type 2 immunity. J. Pathol. 247, 686-696 (2019).

8. Dahlgren, M. W. et al. Adventitial stromal cells define group 2 innate lymphoid cell tissue niches. Immunity 50, 707-722 (2019). 